\title{
Bifurcation and chaos of a flag in an inviscid flow
}

\author{
Ming Chen ${ }^{1}$, Lai-Bing Jia ${ }^{1}$, Yan-Feng Wu ${ }^{1}$, Xie-Zhen Yin ${ }^{1 *}$, Yan-Bao Ma ${ }^{2}$ \\ 1 Department of Modern Mechanics, University of Science and Technology of China, \\ Hefei, Anhui 230027, P.R.China \\ 2 University of California, Merced, Merced, California 95343, USA \\ *Email address: xzyin@ustc.edu.cn
}

\begin{abstract}
A two-dimensional model is developed to study the flutter instability of a flag immersed in an inviscid flow. Two dimensionless parameters governing the system are the structure-to-fluid mass ratio $M^{*}$ and the dimensionless incoming flow velocity $U^{*}$. A transition from a static steady state to a chaotic state is investigated at a fixed $M^{*}=1$ with increasing $U^{*}$. Five single-frequency periodic flapping states are identified along the route, including four symmetrical oscillation states and one asymmetrical oscillation state. For the symmetrical states, the oscillation frequency increases with the increase of $U^{*}$, and the drag force on the flag changes linearly with the Strouhal number. Chaotic states are observed when $U^{*}$ is relatively large. Three chaotic windows are observed along the route. In addition, the system transitions from one periodic state to another through either period-doubling bifurcations or quasi-periodic bifurcations, and it transitions from a periodic state to a chaotic state through quasi-periodic bifurcations.
\end{abstract}

Keywords: Fluid-structure interactions; Flutter instability; Bifurcation; Chaos

\section{Introduction}

Complex interactions of flexible plates with ambient fluid are common in nature, e.g. a flag flapping in the wind and a tree leaf falling in the air. In industry, the flutter of paper has been widely observed in printing processes (Watanabe et al., 2002). In biomedicine, snoring is the result of flow-induced vibration of the soft palate (Aurégan and Depollier, 1995; Huang, 1995).

Due to its practical and theoretical significance, the plate-fluid interaction problem has been studied extensively both experimentally and theoretically. 
Experimental observations of flag flutter have been conducted in soap films (Zhang et al. 2000, Jia et al., 2007; Ristroph and Zhang, 2008), water tunnels (Shelley et al, 2005) and low-speed wind tunnels (Taneda, 1968; Datta and Gottenberg, 1975). By placing a filament in a flowing soap film, Zhang et al. (2000) studied the dynamics of a filament immersed in two-dimensional flow. Two distinct states were found: one is a static stable state for a short filament, and the other is periodic flapping state for a sufficiently long filament. Watanabe et al. (2002) experimentally investigated the dynamics of a sheet in a low-speed wind tunnel. The results showed that the two key factors determining the flutter are the dimensionless bending stiffness of the sheet and the mass ratio between the fluid and the structure. Ait Abderrahmane et al. (2012) experimentally studied the flapping dynamics of a flag in relatively high turbulent flow. Periodic, quasi-periodic and chaotic vibrations were observed in the study. When Shelley et al. (2005) studied the dynamics of a heavy flag interacting with flowing water, it was found that the compliant sheet begins to flap with a Strouhal frequency consistent with animal locomotion. The subcritical Hopf bifurcation was reported separately in these experimental studies. Eloy et al. (2012) showed experimentally that inevitable planeity defects of the flags lead to large hysteresis in the experiments. The influence of the flag shape was experimentally and theoretically studied (Eloy et al., 2007; Eloy et al., 2008), and the results showed that the two-dimensional limit cannot be achieved experimentally because of gravity and 3D effects. The authors also addressed the importance of the flag aspect ratio while predicting the instability threshold.

Different methods have been proposed to numerically simulate the flag-like problem. The most widely used method is the Immersed Boundary (IB) method. Using this method, Zhu and Peskin (2002) studied the dynamics of a filament immersed in a flowing soap film. In the study, it was found that the increase of the mass or length of the filament destabilizes the system. Huang et al. (2007) presented an improved IB method. In their study two boundary conditions at the fixed end, i.e. simply supported or clamped, were considered, and it was found that the amplitude of the oscillating filament with the clamped boundary condition is smaller than that with the simply supported boundary condition. Connell and Yue (2007) proposed a fluid-structure direct simulation (FSDS) method to study the flag flapping problem. They identified three distinct states within a range of mass ratio: the static stable state, 
the period-one limit-cycle flapping state, and the chaotic flapping state. At large Reynolds numbers both the IB method and FSDS method become prohibitively expensive because of the need of very fine grids in the flow field. For the case of an infinite Reynolds number, the panel method has been used to simulate the inviscid and incompressible fluid (Tang et al., 2009a; Tang et al., 2009b). By using the panel method to solve the hydrodynamic pressure and the Galerkin method to solve the dynamics of the flag, Tang and Païdoussis (2007) investigated the post-critical behavior of the flutter system. They found that the critical flow velocity is sensitive to the length of the flag when the flag is short, while it is almost invariant when the flag is sufficiently long. Several periodic flapping states had been observed with the increase of the mass ratio. Alben and Shelley (2008) studied the nonlinear behavior of a two-dimensional flutter system. With the decrease of the dimensionless rigidity, four states were observed in their study: the static stable state, the lower-frequency periodic state, the higher-frequency periodic state, and the chaotic state. A two-dimensional unsteady vortex model based on the Brown-Michael equation was proposed by Michelin et al. (2008). They also carried out a proper orthogonal decomposition (POD) analysis, and it was found that the deformation wave of the flag travels in the streamwise direction, and the forces in the flag travel in the upstream direction.

The chaotic state has been reported in some numerical studies (Connell and Yue, 2007; Alben and Shelley, 2008; Michelin et al., 2008) and experimental studies (Ait Abderrahmane et al., 2011; Ait Abderrahmane et al., 2012). But until now the route to chaos has remained unclear. In the present paper, we develop a two-dimensional model to study the dynamics of a flag immersed in an inviscid flow, and examine the route from a static steady state to a chaotic state with the increase of the dimensionless incoming flow velocity. This paper is organized as follows: a model of the coupled fluid-structure system is proposed in $\S 2$ and validated in $\S 3$; the route to a chaotic state with the increase of the dimensionless incoming flow velocity is presented in $\S 4$; and a summary is given in $\$ 5$.

\section{Theoretical Model and Numerical Method}

\subsection{Problem formulation}

A flag with a high height to width ratio is often considered to be two-dimensional 
(Eloy et al., 2007; Doaré et al., 2011). Here we considered a model of a two-dimensional flag immersed in flow. In the model, the centerline of the flag is assumed to be inextensible. As shown in Fig. 1a, the flag is clamped at the leading edge and free at the trailing edge. The length of the flag is $L$, the mass per unit length of the flag is $\rho_{s}$, the second moment of area is $I$, and the Young's modulus is $E$. The mass per unit area of the fluid is $\rho_{f}$ and the undisturbed incoming flow velocity is $U$.

As shown in Fig. 1, the governing equations of the flag are derived in a local coordinate system. The local tangential direction is denoted by $\vec{\tau}$ and the local normal direction is denoted by $\vec{n}$. According to Newton's 2nd law, the balance of forces on a segment of the flag (Fig. 1b) yields

$$
\frac{d \vec{F}^{i}}{d s}+\vec{f}^{e}-\frac{d}{d t}\left(\rho_{s} \vec{V}\right)=0
$$

where, $s$ is the distance along the flag from the fixed end, $t$ is the time, $\vec{F}^{i}$ is the internal force of the flag, $\vec{f}^{e}$ is the ambient fluid stress acting on the flag, and $\vec{V}$ is the velocity of the flag. The internal force $\vec{F}^{i}$ includes two components: the tension force in the tangential direction $T$ and the shear force in the normal direction $Q$.

(a)

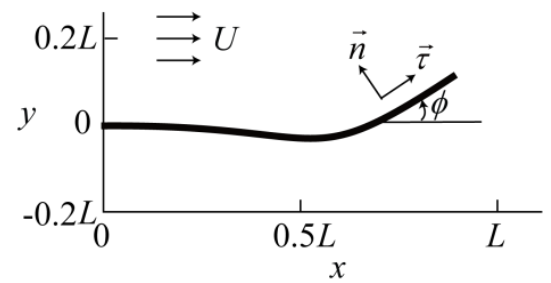

(b)

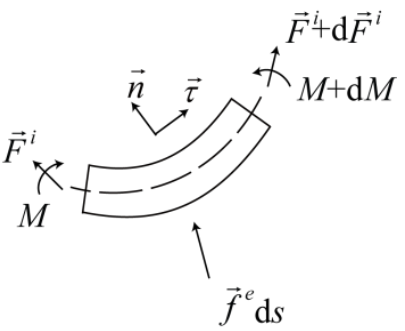

Fig. 1. (a) Description of the problem: a two-dimensional flag immersed in a flow. (b) Forces and moments acting on a segment of the flag.

Assuming the flag is sufficiently thin, the rotational inertia term is of lower order than the terms due to the shear force and the bending (Connell, 2006; Connell and Yue, 2007). As a result, a summation of the moments acting on the segment yields

$$
d M+Q d s=0
$$

where $M$ is the bending moment due to the curvature. The compatibility equation can be established by requiring continuity of the position of the flag in both space and time, written as 


$$
\frac{d}{d t}\left(\frac{d}{d s} \vec{R}(s, t)\right)=\frac{d}{d s}\left(\frac{d}{d t} \vec{R}(s, t)\right)
$$

where $\vec{R}(s, t)$ is a vector to a point on the flag.

Expanding Eqs. (1), (2) and (3) in the tangential and normal directions, and substituting $\partial \phi / \partial s=\Omega$, the governing equations become

$$
\begin{aligned}
\frac{\partial T}{\partial s}-Q \frac{\partial \phi}{\partial s}-\rho_{s} \frac{\partial u}{\partial t}+\rho_{s} v \frac{\partial \phi}{\partial t}+p_{\tau} & =0 \\
\frac{\partial Q}{\partial s}+T \frac{\partial \phi}{\partial s}-\rho_{s} \frac{\partial v}{\partial t}-\rho_{s} u \frac{\partial \phi}{\partial t}+p_{n} & =0 \\
E I \frac{\partial \Omega}{\partial s}+Q & =0 \\
\frac{\partial u}{\partial s}-v \frac{\partial \phi}{\partial s} & =0 \\
\frac{\partial v}{\partial s}+u \frac{\partial \phi}{\partial s}-\frac{\partial \phi}{\partial t} & =0 \\
\frac{\partial \phi}{\partial s}-\Omega & =0
\end{aligned}
$$

where $p_{\tau}$ is the viscous shear stress of the fluid in the tangential direction, $p_{n}$ is the hydrodynamic pressure difference across the flag in the normal direction, $u$ is the tangential velocity component of the flag, $v$ is the normal velocity component of the flag, $\phi$ is the inclination of the local tangential direction away from the direction of the incoming flow, and $\Omega$ is the curvature of the flag. $p_{\tau}$ and $p_{n}$ are calculated by the fluid solver. The six unknown variables are $Y=\left[\begin{array}{llllll}T & Q & u & v & \phi & \Omega\end{array}\right]^{\mathrm{T}}$. Eqs. (4) are similar to the governing equations of the ocean cable used by Gobat and Grosenbaugh (2006), except that the centerline of the flag is inextensible in our model. The boundary conditions at the fixed end are implemented by setting the inclination $\phi$, the normal velocity $u$ and the tangential velocity $v$ to zero, written as

$$
\phi=0, \quad u=0, \quad v=0 .
$$

The boundary conditions at the free end are implemented by setting the curvature, the tension force and the shear force to zero, written as

$$
\Omega=0, \quad T=0, \quad Q=0 .
$$

As the governing equations of the flag are represented in a curvilinear coordinate system, we need to build the relationship between the Cartesian coordinates and the 
curvilinear coordinates. As shown in Fig. 1a, the origin of the Cartesian coordinate system is set at the clamped leading edge of the flag, the $x$ axis is along the free stream, and the $y$ axis is normal to the free stream direction from bottom to top. The flag positions in the $x-y$ coordinate system are calculated by the integration of the following equations:

$$
\begin{aligned}
& \frac{\partial x}{\partial s}=\cos \phi \\
& \frac{\partial y}{\partial s}=\sin \phi
\end{aligned}
$$

Scaling lengths on $L$, time on $L / U$, and mass on $\rho_{f} L^{2}$, the governing equations (4) are nondimensionalized as follows:

$$
\begin{aligned}
\frac{\partial \hat{T}}{\partial \hat{s}}-\hat{Q} \frac{\partial \phi}{\partial \hat{s}}-M^{*} \frac{\partial \hat{u}}{\partial \hat{t}}+M^{*} \hat{v} \frac{\partial \hat{\phi}}{\partial \hat{t}}+\hat{p}_{\tau} & =0, \\
\frac{\partial \hat{Q}}{\partial \hat{s}}+\hat{T} \frac{\partial \phi}{\partial \hat{s}}-M^{*} \frac{\partial \hat{v}}{\partial \hat{t}}-M^{*} \hat{u} \frac{\partial \phi}{\partial \hat{t}}+\hat{p}_{n} & =0, \\
\left(\frac{1}{U^{*}}\right)^{2} \frac{\partial \hat{\Omega}}{\partial \hat{s}}+\hat{Q} & =0, \\
\frac{\partial \hat{u}}{\partial \hat{s}}-\hat{v} \frac{\partial \hat{\phi}}{\partial \hat{s}} & =0, \\
\frac{\partial \hat{v}}{\partial \hat{s}}+\hat{u} \frac{\partial \phi}{\partial \hat{s}}-\frac{\partial \phi}{\partial \hat{t}} & =0, \\
\frac{\partial \phi}{\partial \hat{s}}-\hat{\Omega} & =0 .
\end{aligned}
$$

where $\hat{p}_{\tau}$ and $\hat{p}_{n}$ are the dimensionless fluid stresses in the tangential direction and the normal direction, respectively, and they are written as

$$
\begin{aligned}
& \hat{p}_{\tau}=\frac{p_{\tau}}{\rho_{f} U^{2}}, \\
& \hat{p}_{n}=\frac{p_{n}}{\rho_{f} U^{2}} .
\end{aligned}
$$

The six dimensionless unknown variables are $\hat{Y}=\left[\begin{array}{llllll}\hat{T} & \hat{Q} & \hat{u} & \hat{v} & \hat{\phi} & \hat{\Omega}\end{array}\right]^{\mathrm{T}}$. The two dimensionless governing parameters of the system are

(1) $M^{*}=\rho_{s} / \rho_{f} L$, the structure-to-fluid mass ratio,

(2) $U^{*}=U\left(\rho_{f} L^{3} / E I\right)^{1 / 2}$, the dimensionless incoming flow velocity. 
The dimensionless Cartesian coordinate system is represented by $(X, Y)$.

Eqs. (8) are solved by a finite difference method. As shown in Fig. 2, the flag is divided into $N$ panels. The node indices start from the clamped point $(i=1)$ and end at the trailing end $(i=N+1)$. The mesh spacing is denoted by $\Delta \hat{s}$ and the time step size by $\Delta \hat{t}$. The governing equations of the flag are discretized in space on half-grid points with second-order accuracy. Denoting an arbitrary variable as $\varphi$, at the $j$ th time step, the discretization of the first-order spatial derivative at $(i-1 / 2) \Delta \hat{s}$ is expressed as

$$
{\frac{\partial \varphi^{j}}{\partial \hat{s}_{i-1 / 2}}}^{j} \frac{\varphi_{i}^{j}-\varphi_{i-1}^{j}}{\Delta \hat{s}}
$$

The first-order temporal derivative is calculated with first-order accuracy, expressed as

$$
{\frac{\partial \varphi^{j}}{\partial \hat{t}_{i-1 / 2}}}^{j}=\frac{\varphi_{i-1 / 2}^{j}-\varphi_{i-1 / 2}^{j-1}}{\Delta \hat{t}}=\frac{\left(\varphi_{i-1}^{j}+\varphi_{i}^{j}\right)-\left(\varphi_{i-1}^{j-1}+\varphi_{i}^{j-1}\right)}{2 \Delta \hat{t}} .
$$

An iterative Newton-Raphson scheme is used to solve the discretized equations (Ypma, 1995).

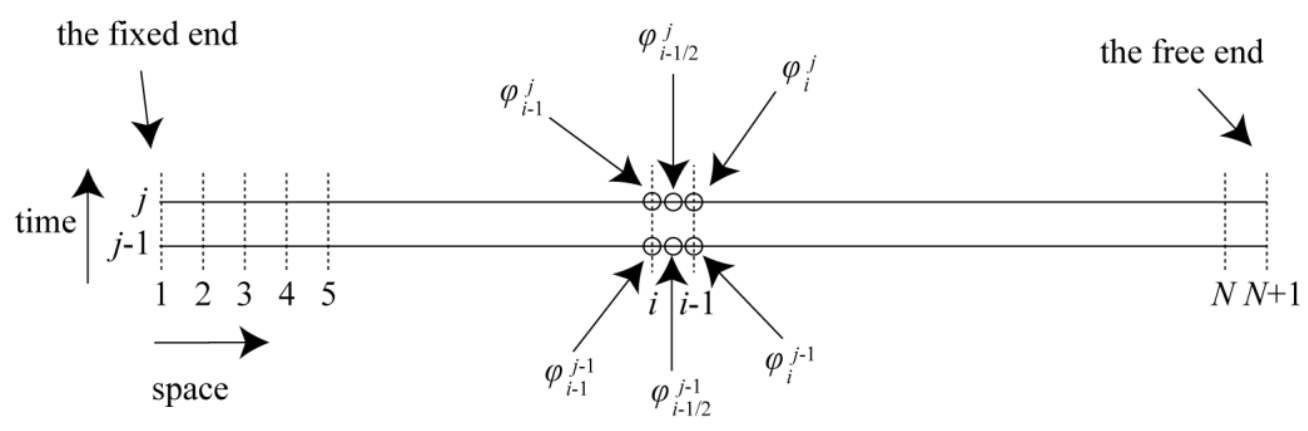

Fig. 2. Schematic diagram of the discretization of the governing equations of the flag.

\subsection{Calculation of the pressure difference}

The fluid is assumed to be inviscid and incompressible, and a panel method is used to calculate the pressure on the flag (Tang and Païdoussis, 2007; Wang and Yin, 2010). As shown in Fig. 3a, the flag is divided into $N$ panels, and the panels are numbered from the fixed end. As shown in Fig. 3b, for the $i$ th panel at the $j$ th 
time-step, a lumped vortex $\left(\Gamma_{i}^{j}, 1 \leq i \leq N\right)$ is placed in the first quarter of the panel, and a collection point $\left(C_{i}^{j}, 1 \leq i \leq N\right)$ is placed in the third quarter of the panel. At the $j$ th time-step, a new vortex $\Gamma_{w}^{j}$ sheds from the tail. These wake vortices travel at local flow velocities, and the strengths of the free vortices in the wake remain unchanged in time. The strength of the bound vortex $\Gamma_{i}^{j}$ is denoted by $\gamma_{i}^{j}$, and the strength of the wake vortex $\Gamma_{w}{ }^{j}$ by $\gamma_{w}{ }^{j}$. The potential $\Theta$ at $\left(x_{1}, y_{1}\right)$ induced by a lumped vortex of the strength $\gamma$ located at $\left(x_{2}, y_{2}\right)$ is written as

$$
\Theta\left(\gamma, x_{1}, y_{1}, x_{2}, y_{2}\right)=-\frac{\gamma}{2 \pi} \arctan \frac{y_{1}-y_{2}}{x_{1}-x_{2}}
$$

The fluid velocity $\vec{V}_{f}$ is given by

$$
\vec{V}_{f}=\nabla\left(U x+\Theta_{f}\right)
$$

where $\Theta_{f}$ is the sum of the potential induced by all the vortices in the fluid.

At the $j$ th time-step, $N+1$ variables are unknown in the fluid solver, i.e. the strengths of bounded vortices $\gamma_{i}^{j}(1 \leq i \leq N)$ and the free vortex shed at the current time-step $\gamma_{w}{ }^{j}$. Thus $N+1$ equations are needed to solve for these unknown variables. Here we apply the no-penetration condition at the $N$ collection points and the Kelvin's theorem. The no-penetration condition implies that on each collection point the fluid velocity in the normal direction is equal to the velocity of the flag in the normal direction, i.e.

$$
\vec{V}_{f} \cdot \vec{n}=v
$$

The Kelvin theorem implies that the total vorticity in the fluid is zero, i.e.

$$
\sum_{i=1}^{N} \gamma_{i}^{j}+\sum_{k=1}^{j} \gamma_{w}^{j}=0
$$

Generally the Kutta condition is also needed to guarantee that the velocity remains finite along the sharp trailing edge (Hess, 1990). In our model the Kutta condition is approximately satisfied by specifying the bound vortex on the first quarter of each panel and the collection point on the third quarter (Katz and Plotkin, 2001). The same method has also been used by Tang and Païdoussis (2007). 
As the fluid is assumed to be inviscid, the shear stress in the tangential direction $p_{\tau}$ is zero. The pressure difference in the normal direction $p_{n}$ on the $i$ th collection point at the $j$ th time step is calculated using the unsteady Bernoulli equation (Alben, 2008; Alben, 2012), i.e.

$$
p_{n}=\rho_{f}\left[\frac{\left(\vec{V}_{f}^{+}+\vec{V}_{f}^{-}\right)}{2} \cdot \vec{\tau}-u\right] \frac{\gamma_{i}^{j}}{\Delta s}+\rho_{f} \sum_{k=1}^{i} \frac{\partial \gamma_{k}^{j}}{\partial t}
$$

where $\vec{V}_{f}^{+}$and $\vec{V}_{f}^{-}$are the fluid velocities on the upside and downside of the flag, respectively, and $\Delta s=L / N$ is the length of an individual panel.

(a)

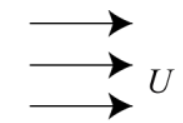

1st panel 2nd panel

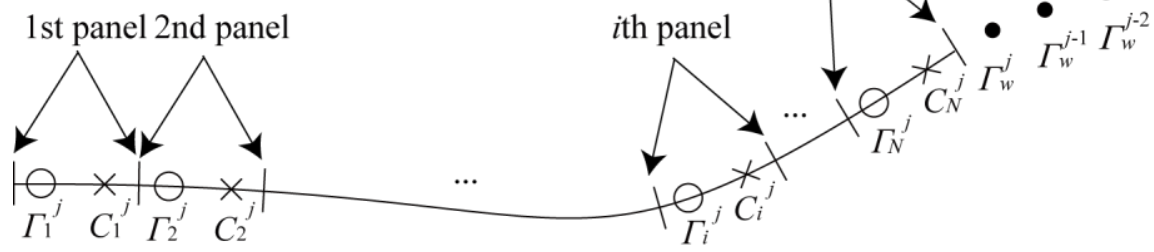

(b)

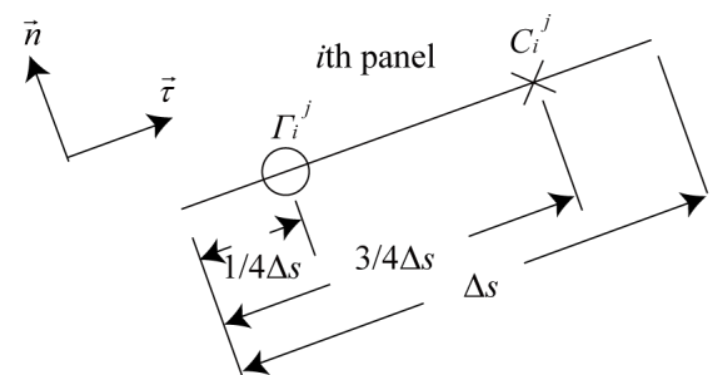

Fig. 3. Schematic diagram of the panel method. (a) Distribution of the bound vortices (open circles), collection points (crosses) and free wake vortices (solid circles). (b) An individual panel with a bound vortex and a collection point.

\subsection{The iterative procedure between the structure solver and the fluid solver}

As the coupled nonlinear problem requires force equilibrium at the structure-fluid interface at each time step, an iterative approach between the structure solver and the fluid solver is used to solve the problem. At the $(n+1)$ th time step, in the first loop a predicted $\hat{Y}_{n+1}$ is calculated in the form of $\hat{Y}_{n+1}=2 \hat{Y}_{n}-\hat{Y}_{n-1}$, then a 
pressure distribution is obtained by solving Eq. (16) with the predicted $\hat{Y}_{n+1}$. In the second loop, the predicted $\hat{Y}_{n+1}$ is updated by solving Eq. (8) with the obtained pressure distribution, and again, the pressure distribution is recalculated with the current $\hat{Y}_{n+1}$. The iterative loop continues until the system has converged to a solution.

\section{Validation}

In the simulation, the mesh spacing $\Delta \hat{s}$ is 0.01 and the time step size $\Delta \hat{t}$ is 0.005. All cases start with the same initial condition, where the curvature of the flag $\hat{\Omega}$ is set to be constant to keep the tail displacement at 0.02 . The calculation time is long enough for the full development of the initial displacement disturbance. In the simulation, depending on $U^{*}$, the system with a specified $M^{*}$ has two possible states, i.e. the flag keeps align with the incoming flow, or the flag flaps. Fig. 5 plots the stability boundary of the system as a function of $M^{*}$, where the solid line with filled squares denotes our simulation results, and the dashed line denotes the stability boundary obtained by Watanabe et al. (2002a). Obviously these two results agree well.

Experiments were carried out in a low-speed wind tunnel to validate the simulation. As shown in Fig. 4, the cross-section of the wind tunnel is $1 \mathrm{~m} \times 1 \mathrm{~m}$. The velocity of the incoming flow was measured with a hot-wire anemometry (TSI-8384-M-GB). The flow velocity in the test section ranges from $3 \mathrm{~m} / \mathrm{s}$ to $50 \mathrm{~m} / \mathrm{s}$. A light sheet from a semiconductor laser was used to illuminate the flag. A high-speed digital video camera (Mikrotron, MC1310) was used to record the movements of the flag at 1000 frames per second. To reduce the disturbance from the strut, two steel wires with a diameter of $1 \times 10^{-4} \mathrm{~m}$ were used to fix the flag vertically in the central region of the test region. The rectangular flag was cut from a flexible Mylar sheet. The mass of the Mylar sheet per unit area is $0.136 \mathrm{~kg} / \mathrm{m}^{2}$ and the bending stiffness of the Mylar sheet per unit span is $4.989 \times 10^{-4} \mathrm{~N} \mathrm{~m}$. The height to width ratio of the flag in the experiment is 1 . For more details about the experimental setup, please see Pang et al. (2010). In the experiment, the flow velocity was slowly increased from zero. At small velocities, the system is in a static stable state. When the velocity is above a critical value $U_{c}^{*}$, the flag flaps spontaneously. Due to the limitation of the flag 
material, the velocity of the incoming fluid was increased no more than $16 \mathrm{~m} / \mathrm{s}$. Then the flow velocity was slowly decreased. When the velocity is below another critical value $U_{d}^{*}$, the system returns to its static stable state.

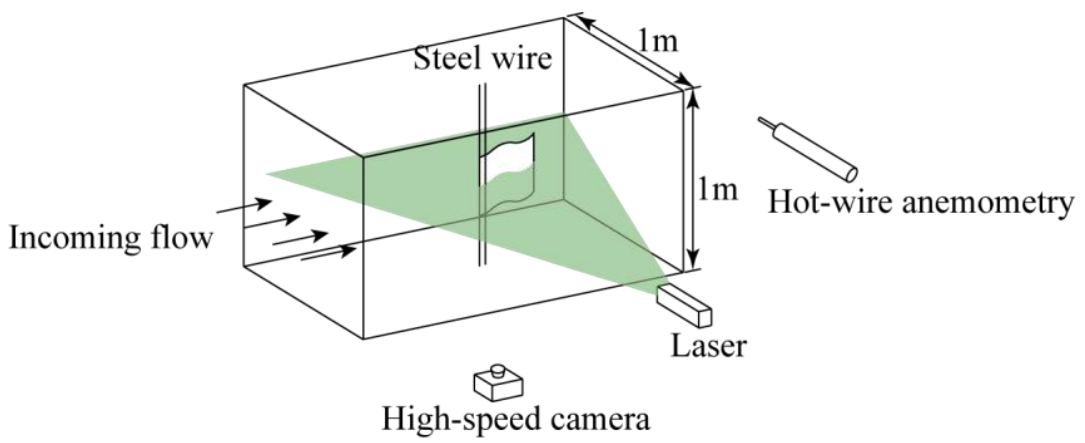

Fig. 4. Sketch of the experimental setup.

In Fig. 5 the filled up triangles and filled down triangles correspond to $U_{c}^{*}$ and $U_{d}^{*}$ in our experiment, respectively. The crosses are $U_{c}^{*}$ from Huang (1995), the open up triangles and open circles are $U_{c}^{*}$ from Eloy et al. (2008), and the open sqrares are $U_{c}^{*}$ from Schouveiler and Eloy (2009). As shown in Fig. 5, the critical flow velocities in experiments are higher than those in simulations, which implies that the $3 \mathrm{D}$ model in experiments is more stable than the $2 \mathrm{D}$ model in simulations. This is consistent with Eloy et al. (2008) and Doaré et al. (2011). As a validation, in Fig. 6 the snapshots of the flag for $M^{*}=1$ in our simulation (Fig. 6a-f) are compared with those in our experiment (Fig. 6g-1).

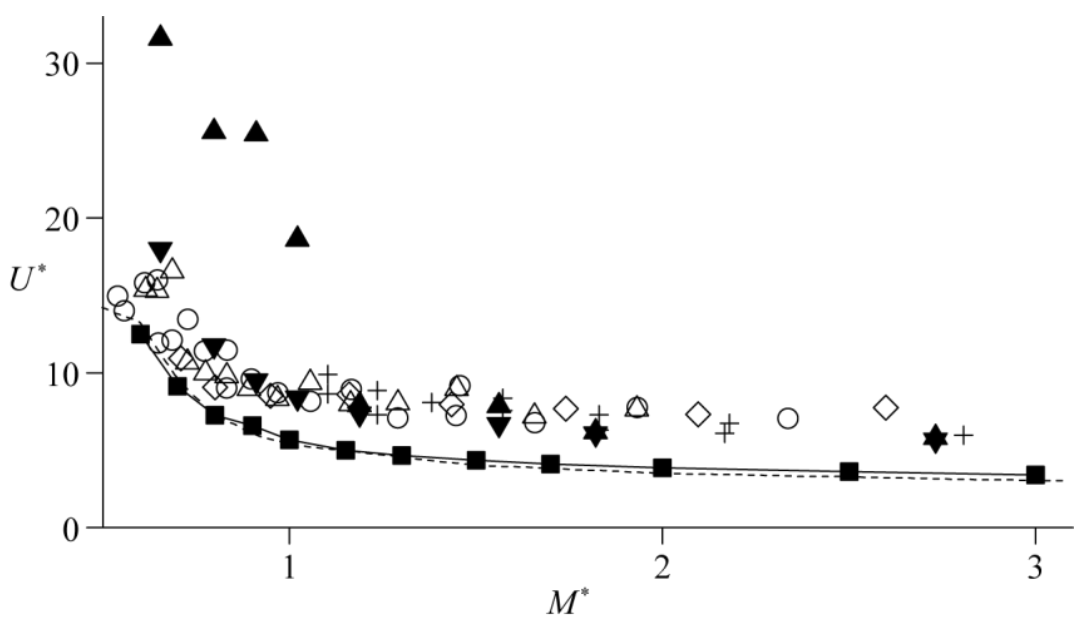

Fig. 5. The critical velocity versus the structure-to-fluid mass ratio $M^{*}$. The solid line 
with filled squares denotes the stability boundary in our simulation. The dashed line is the stability boundary from Watanabe et al. (2002a). The crosses are $U_{c}^{*}$ from Huang (1995), the open up triangles and open circles are $U_{c}^{*}$ from Eloy et al. (2008), and the open sqrares are $U_{c}^{*}$ from Schouveiler and Eloy (2009). The filled up triangles and filled down triangles correspond to $U_{c}^{*}$ and $U_{d}^{*}$ in our experiment, respectively.
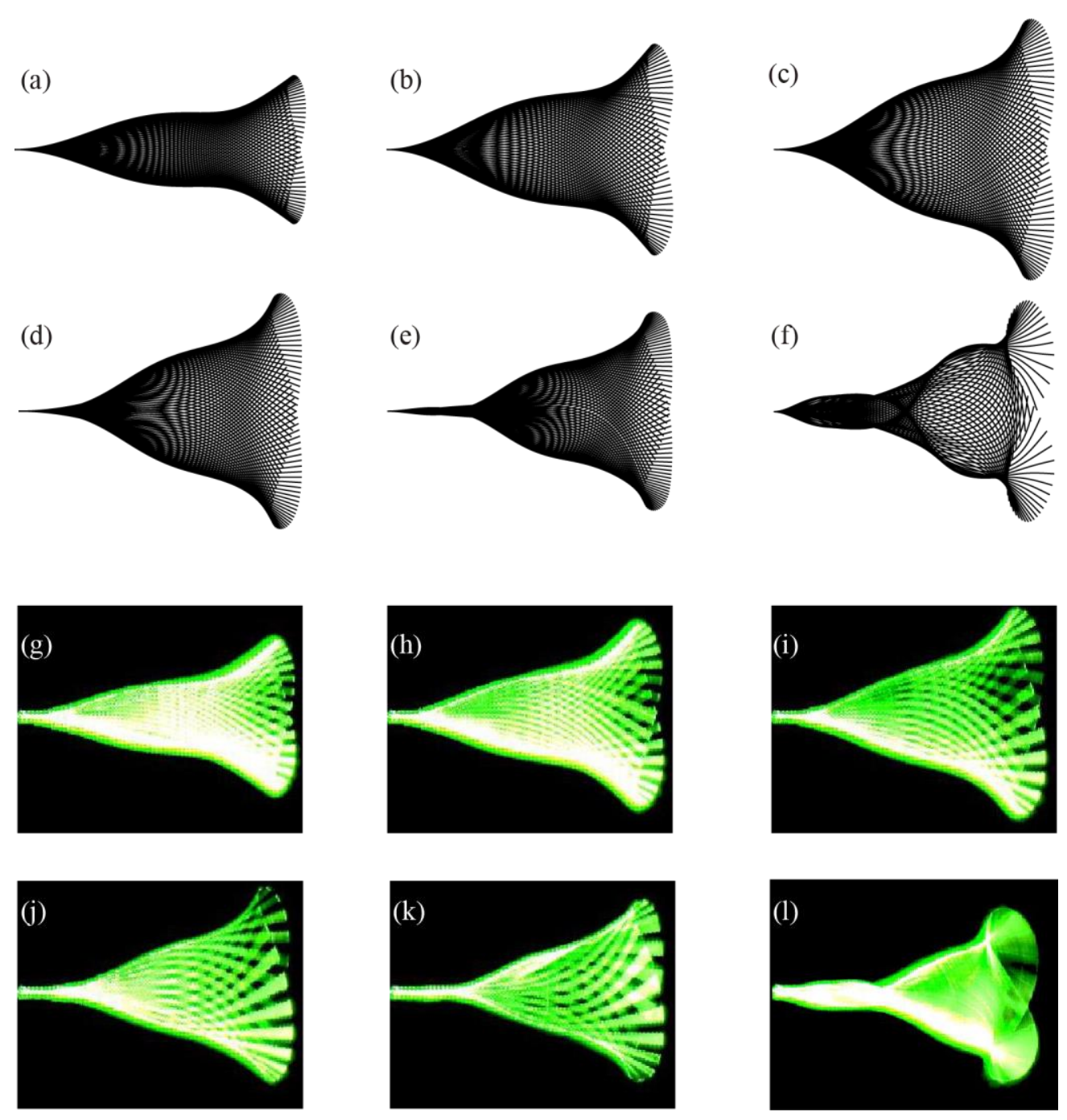

Fig. 6. The variation of the flag snapshot with the increase of $U^{*}$ in our simulation (a-f) and in our experiment (g-l). For all the cases $M^{*}=1$. In the simulation, $U^{*}$ is (a) 6.6, (b) 7.3, (c) 9.5, (d) 12.0, (e) 13.1, (f) 15.8. In the experiment, $U^{*}$ is (g) 9.1, (h) 10.1, (i) 12.8, (j) 15.1, (k) 16.5 , (l) 25.1 . 


\section{Results and discussion}

\subsection{Route to a chaotic state}

For a system with a specified $M^{*}$, with the increase of $U^{*}$, three states are observed by Alben and Shelley (2008): the static stable state, the periodic flapping states, and the chaotic state. In the present study, we will study the route from a steady state to a chaotic state at a fixed $M^{*}=1$. Compared with the study carried out by Alben and Shelley (2008), in our model $M^{*}$ is bigger and $U^{*}$ covers a wider range. The complex behavior of the system is shown in Fig. 7. Fig. 7a shows the bifurcation diagram, where for each case the maximum displacements of the tail in both the positive and negative directions in each circle are recorded. In Fig. 7a the local amplitude maxima are a single point for a static stable case (region a), two points for a single-frequency periodic case (region $b, d, h, j, n$ ), and four points or multiple of four points for a period-doubling bifurcation case (region i). The local amplitude maxima cover a range for a quasi-periodic bifurcation case (region c, e, g, k, m) (Zhang et al., 2009), and for a chaotic case this range breaks out (region $\mathrm{f}, 1$ and o). The results of a fast Fourier transform (FFT) analysis of the tail displacement for the corresponding cases are shown in Fig. $7 \mathrm{~b}$, where for each case the amplitude of the original FFT results is normalized by the maximum amplitude of this case, and the normalized amplitude of the frequency spectrum is represented by the color. 
(a)

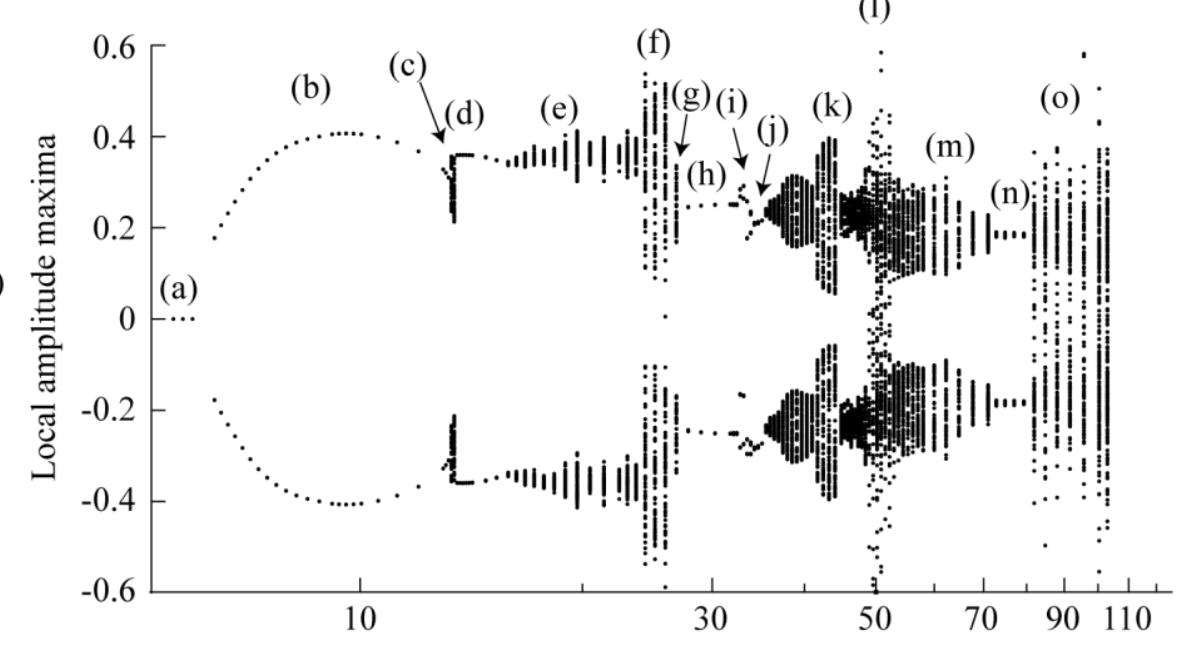

(b) $f$

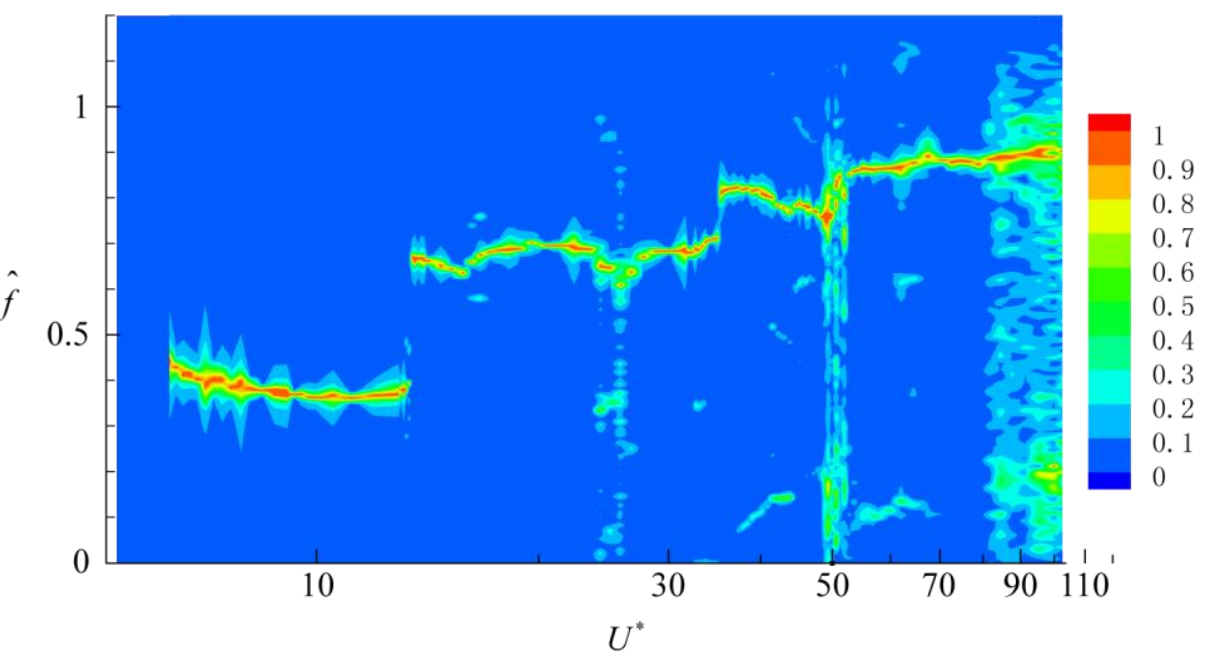

Fig. 7. (a) The bifurcation diagram with the increase of $U^{*}$ for $M^{*}=1$. (b) The corresponding FFT analysis results, where the color represents the normalized amplitude of the frequency spectrum.

Rich dynamics are observed in the transition route shown in Fig. 7. The system is in a static stable state for $U^{*}<5.7$. At $U^{*}=5.7$ the system loses its stability and transitions to a single-frequency periodic state (region b in Fig. 7a) via a Hopf bifurcation. Further increase of $U^{*}$ causes quasi-periodic bifurcations (region c), and then a new periodic state with a much higher frequency is observed (region d). When $U^{*}$ is further increased, a sequence of quasi-periodic bifurcations (region e) are observed, and at $U^{*}=23.6$, a chaotic state (region f) is firstly observed. When $U^{*}$ is further increased, the chaos region is interrupted. Quasi-periodic bifurcations are observed in region $\mathrm{g}$, and again, a new periodic state is found in region $\mathrm{h}$. Period-doubling bifurcations are observed in region $i$, and then a new asymmetric 
oscillation state is found in region $\mathrm{j}$. Via quasi-periodic bifurcations in region $\mathrm{k}$, the system enters another chaotic state (in region 1). Further increase of $U^{*}$ suppresses the chaos and the quasi-periodic bifurcation region $\mathrm{m}$ is found. In region $\mathrm{n}$, the flag flaps periodically again, and when $U^{*}$ is further increased, the system enters the final chaotic state (region o). Finally, the system remains in the chaotic state when $U^{*}$ is further increased.

Five periodic states (including four symmetric states in regions b, d, h, n and one asymmetric state in region $\mathrm{j}$ ) and three chaotic states (in regions $\mathrm{f}, \mathrm{l}, \mathrm{o}$ ) are observed in this route. The four symmetrical single-frequency periodic states will be studied in $\S 4.2$. The transition from one periodic state to another periodic state will be studied in $\S 4.3$. The transition from a periodic state to a chaotic state will be studied in $\S 4.4$.

\subsection{Symmetrical period-one limit cycle oscillation states}

Four symmetrical period-one limit cycle oscillation states are observed in the transition to chaos (regions b, d, h, n in Fig. 7a). The flag snapshots of these four states are shown in the left column of Fig. 8. It is found that with the increase of $U^{*}$, more "necks" appear. The corresponding vorticity in the fluid is shown in right column of Fig. 8, including the bound vorticity on the flag and the free vorticity in the wake. As a result of the increase of the flapping frequency, the distance between two successive vortex structures in the wake decreases with the increase of $U^{*}$. It should be noted that in Fig. 8 the wakes look chaotic with clumping of vortex blobs, which could be regularized by the filtering technique (Krasny, 1986). 
(a)

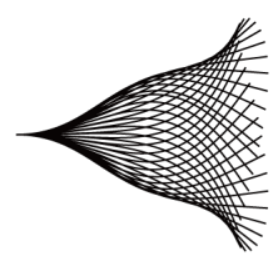

(b)

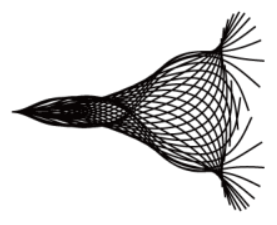

(c)

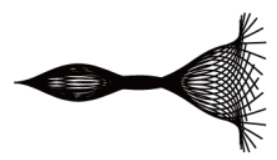

(d)

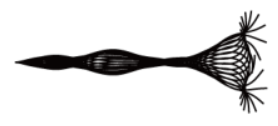

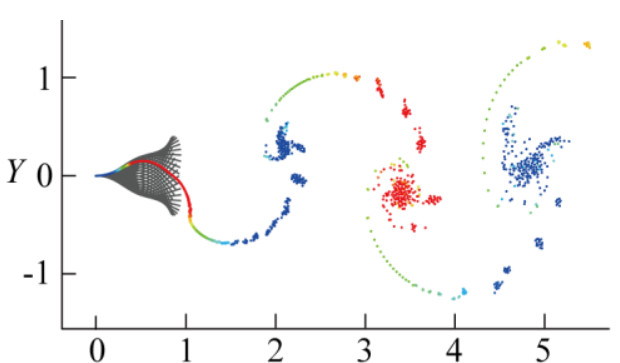
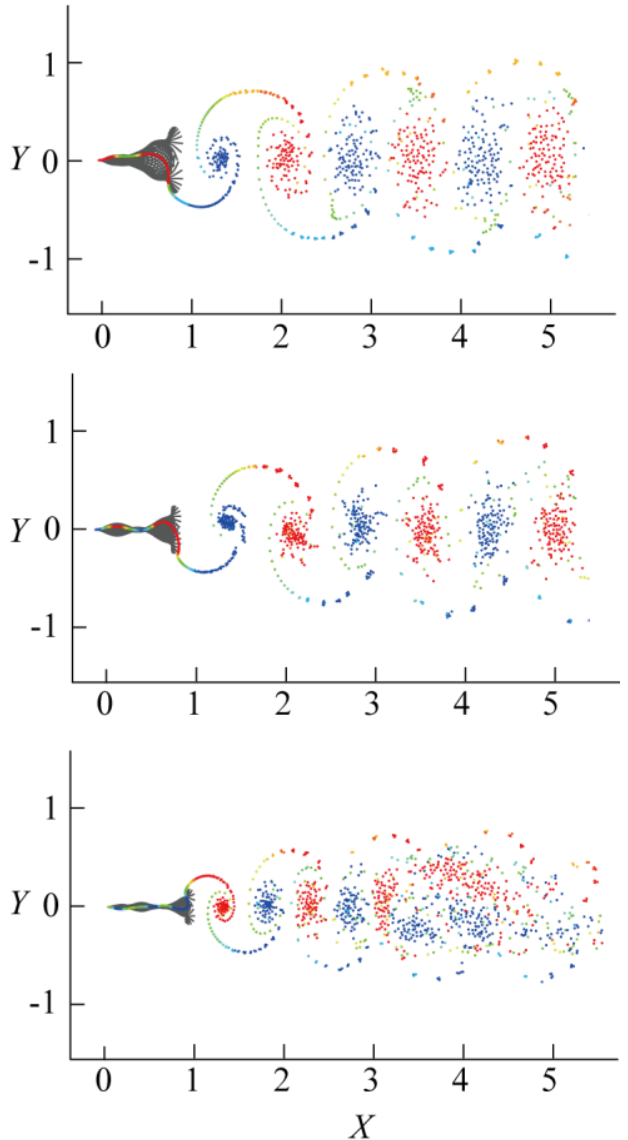

Fig. 8. Snapshots of the periodic flapping flag (left column) and wake structures (right column) in the simulation. For all these cases $M^{*}=1, U^{*}$ is (a) 10, (b) 15.8, (c) 27.7, (d) 76.7 .

Fig. 9 shows the dimensionless frequency $\hat{f}$, the dimensionless amplitude $\hat{A}$ and dimensionless time-averaged drag force $\hat{D}$ for these four states. The dimensionless time-averaged drag force on the flag $\hat{D}$ is defined as

$$
\hat{D}=\hat{f} \int_{t_{0}}^{t_{0}+\hat{f}^{-1}} \int_{0}^{1} \hat{P}_{n} d \hat{s} d \hat{t}
$$

where $t_{0}$ is a time point, and $\hat{P}_{n}$ represents the component of the pressure difference on the flag in the streamwise direction. The dimensionless frequency $\hat{f}$ and the 
dimensionless amplitude $\hat{A}$ for the two periodic flapping states in the experiment are also presented in Fig. 9. $\hat{f}$ and $\hat{A}$ in the experiment are respectively defined as

$$
\begin{aligned}
& \hat{f}=f L / U, \\
& \hat{A}=A / L,
\end{aligned}
$$

where $f$ and $A$ are the frequency and the amplitude in the experiment, respectively. As shown in Fig. 9, the results in the simulation show similar trends as the results in the experiment, except that the transition region between the two periodic states in the experiment (between $U^{*}=16.5$ and $U^{*}=24.8$ ) is larger than that in the simulation (between $U^{*}=13.2$ and $U^{*}=13.4$ ).

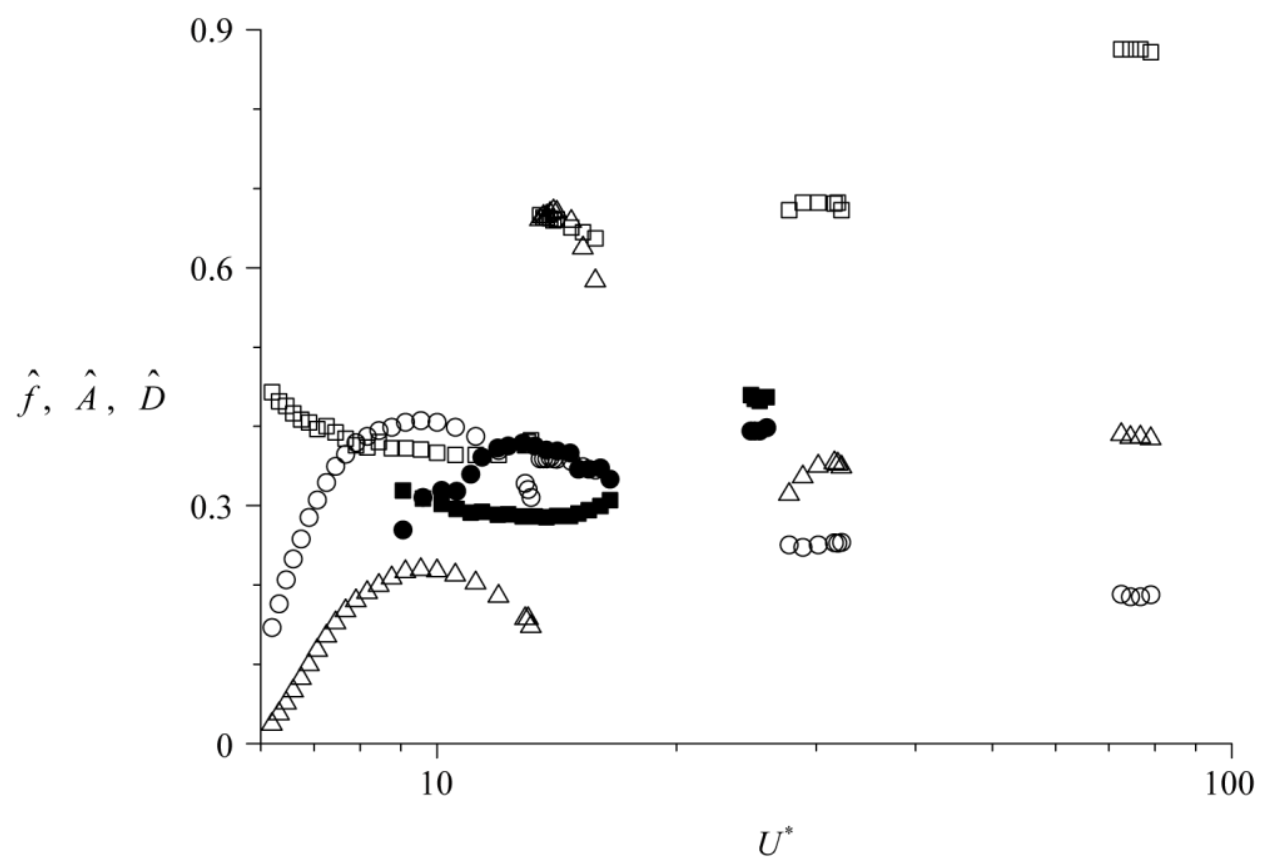

Fig. 9. The dimensionless frequency $\hat{f}$ (squares), the dimensionless amplitude $\hat{A}$ (circles) and the dimensionless drag force $\hat{D}$ (triangles) of the four symmetrical periodic flapping states as a function of the dimensionless incoming flow velocity $U^{*}$ for $M^{*}=1$. The open symbols denote the results in the simulation and the solid symbols denote the results in the experiment.

The force exerted by fluid on a deformable body immersed in flow depends on the kinematic parameters of the system (Alben et al., 2002). To study the drag forces on tandem arranged flags, Ristroph \& Zhang (2008) carried out an experiment in a flowing soap film and found that the drag force on a single flag scales with the 
flapping amplitude. We notice that all the cases in their study are in the first periodic state (Fig. 8a). In order to examine the relationship between the drag force and the kinematic parameters for all the periodic states, in the present study we employ the Pearson product-moment correlation coefficient (Pearson's $r$ ). The Pearson's $r$ is a measure of the correlation between two variables (Rodgers \& Nicewander 1988). For two variables $A$ and $B$, the Pearson's $r$ is defined as

$$
r=\frac{\sum\left(A_{i}-\bar{A}\right)\left(B_{i}-\bar{B}\right)}{\left[\sum\left(A_{i}-\bar{A}\right)^{2} \sum\left(B_{i}-\bar{B}\right)^{2}\right]^{1 / 2}}
$$

where the bar denotes the sample mean. The value of Pearson's $r$ ranges from -1 to 1 . A value 1 implies that $B$ linearly increases with the increase of $A,-1$ implies that $B$ linearly decreases with the increase of $A$, and 0 implies that there is no linear correlation between the two variables. The correlation coefficients between the dimensionless drag $\hat{D}$ and three kinematic parameters, i.e. the dimensionless frequency $\hat{f}$, the dimensionless amplitude $\hat{A}$, and the Strouhal number $S t$, are shown in Table 1. Here $S t$ is defined as

$$
S t=\hat{f} \hat{A} \text {. }
$$

In Table 1 the correlation coefficient between $\hat{D}$ and $S t$ is 0.9761 for all the periodic states, which implies that the dimensionless drag on the flag changes linearly with the Strouhal number. It should be noted that for the first periodic state (Fig. 8a), the correlation coefficient between $\hat{D}$ and $\hat{A}$ is 0.9851 , which implies that the dimensionless drag also changes linearly with the dimensionless amplitude of the flag for the first periodic state.

Table 1 List of the correlation coefficients between the dimensionless drag and different kinematic parameters

\begin{tabular}{cccc}
\hline & $\hat{A}$ & $\hat{f}$ & $S t$ \\
& & & \\
\hline Cases in the first periodic state & 0.9851 & -0.9658 & 0.9638 \\
& & & \\
\hline Cases in all periodic states & 0.2008 & 0.6925 & 0.9761 \\
& & & \\
\hline
\end{tabular}




\subsection{Transition from one periodic state to another periodic state}

As shown in Fig. 7, the five periodic states are isolated from each other. Here we study the transitions between them. Fig. 10 shows the transition from one periodic state (region b) to another higher frequency periodic state (region d) via a quasi-periodic bifurcation (region c). The left column of Fig. 10 plots the time variation of the tail displacement, the middle column shows the corresponding FFT analysis results, and the right column shows the phase plot of the tail velocity in the cross-stream direction against the tail displacement in the cross-stream direction. In Fig. 10a $\left(U^{*}=13.1\right)$, the flag flaps periodically, and the FFT analysis result in the middle column provides the dominant frequency $f_{1}$. When $U^{*}$ is slightly increased to 13.2, as shown in Fig. 10b, a quasi-periodic bifurcation is observed. In the left column of Fig. 10b, the amplitude of the time variation of the tail displacement becomes a sinusoid. In the corresponding spectrum analysis shown in the middle column of Fig. $10 \mathrm{~b}$, the second basic frequency $f_{2}$ cannot be directly observed, but some linear combinations of these two basic frequencies $f_{1}+m f_{2}$ are identified, where $m$ is an integer. As a result of the irreducible second basic frequency $f_{2}$, the phase plot in the right column of Fig. 10b covers a finite range. When $U^{*}$ is further increased to 13.5, as shown in Fig. 10c, the flag flaps periodically again. The FFT analysis in the middle column of Fig. 10c shows that the new dominant frequency $f_{1}$ for $U^{*}=13.5$ is much higher than that for $U^{*}=13.1$ (Fig. 10a). 
(a)
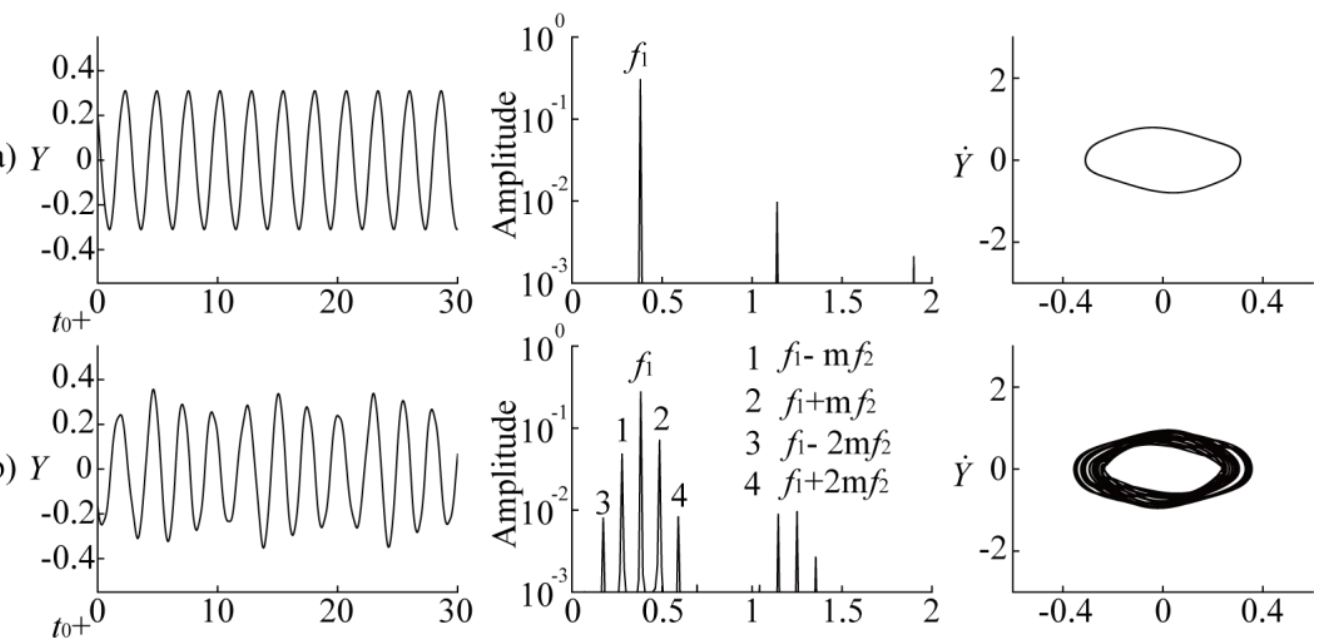

(c)
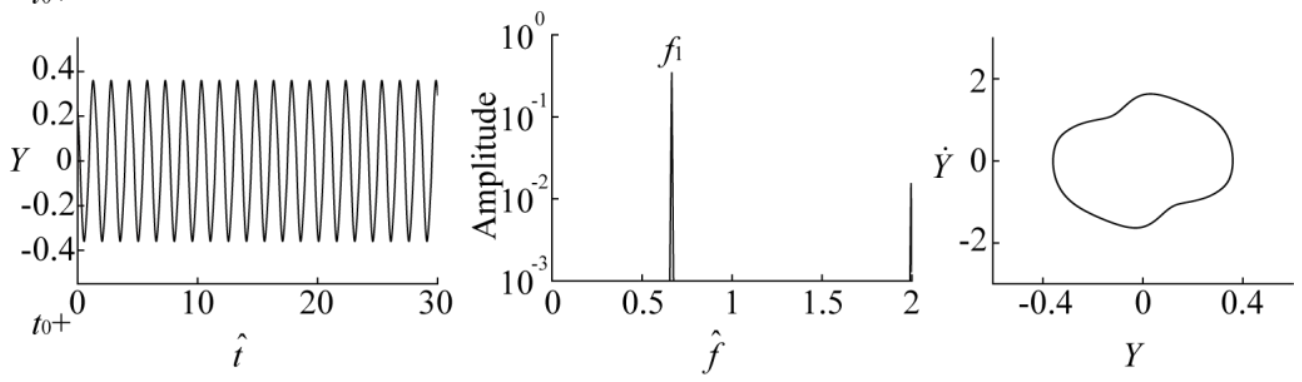

Fig. 10. Transitions from one periodic state to another higher frequency periodic state via quasi-periodic bifurcation. For all the cases $M^{*}=1, U^{*}$ is (a) 13.1 , (b) 13.2 , (c) 13.5. Left column: time variation of the tail displacement. Middle column: FFT analysis of the tail displacement. Right column: phase plot of the tail velocity in the cross-stream direction against the tail displacement in the cross-stream direction.

In the experiment, quasi-periodic bifurcations were also observed when the system transitions from one periodic state to another periodic state. Fig. 11a shows the dimensionless cross-stream displacement plotted against the dimensionless time for $M^{*}=1$ and $U^{*}=21.2$. The time variation of the tail displacement in this transition region observed in the experiments shown in Fig. 11a is similar to the simulation results shown in the left column of Fig. 10b. Fig. 11b shows the phase plot of the dimensionless along-steam tail displacement against the dimensionless cross-stream tail displacement. 
(a)

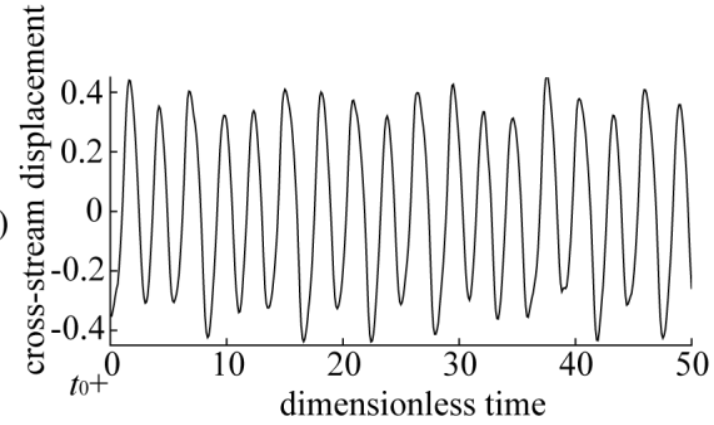

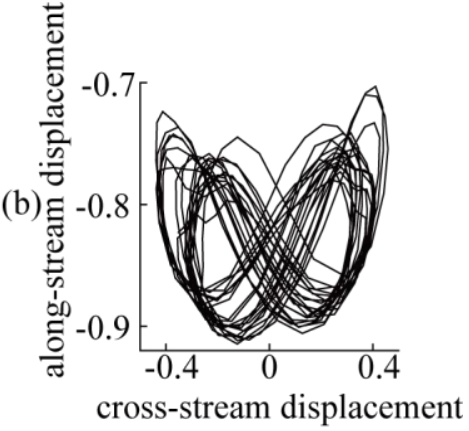

Fig. 11. Experimental results in the transition region for $M^{*}=1$ and $U^{*}=21.2$. (a) The dimensionless cross-steam displacement of the tail plotted against the dimensionless time. (b) The dimensionless along-stream displacement of the tail plotted against the dimensionless cross-stream displacement of tail.

Fig. 12 shows the transition from a symmetrical periodic state (region $\mathrm{h}$ ) to an asymmetrical periodic state (region $\mathrm{j}$ ) via a period-doubling bifurcation (region i). As shown in Fig. 12a, the system is in a symmetrical periodic state when $U^{*}$ is 32.3. When $U^{*}$ is slightly increased to 33.3, as shown in Fig. 12b, a period-doubling bifurcation is observed. The FFT analysis shown in the middle column of Fig. 12b clearly shows the subharmonic frequency $f_{1} / 2$. As a result of the subharmonic frequency, the phase plot shown in the right column of Fig. 12b has two orbits. When $U^{*}$ is increased further to 34.1, as shown in Fig. 12c, the bifurcation is suppressed, and another period-one limit cycle state is observed. However, unlike the symmetrical state shown in Fig. 12a, the oscillation in this state is asymmetrical. The snapshot of the flag and the wake structure of the asymmetrical state are shown in Fig. 13. 
(a)

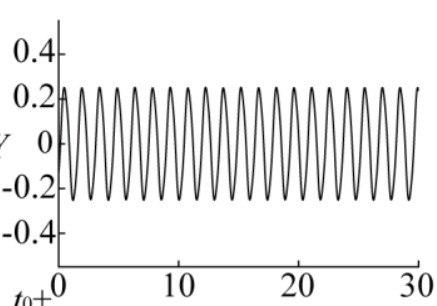

(b) $Y$

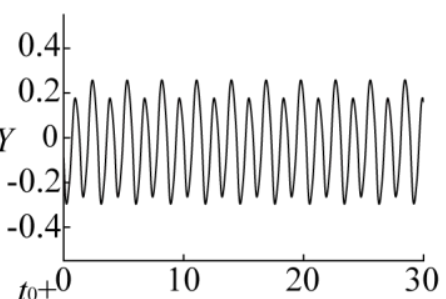

(c)

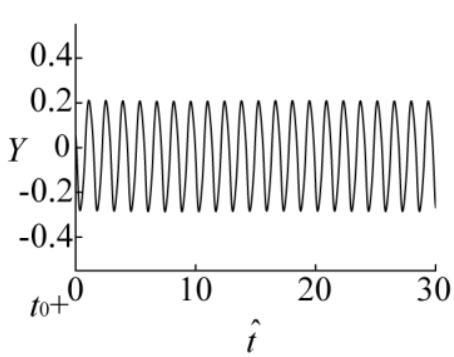

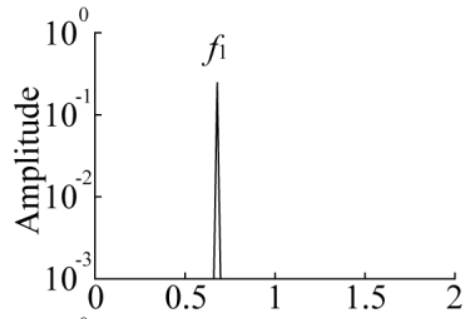
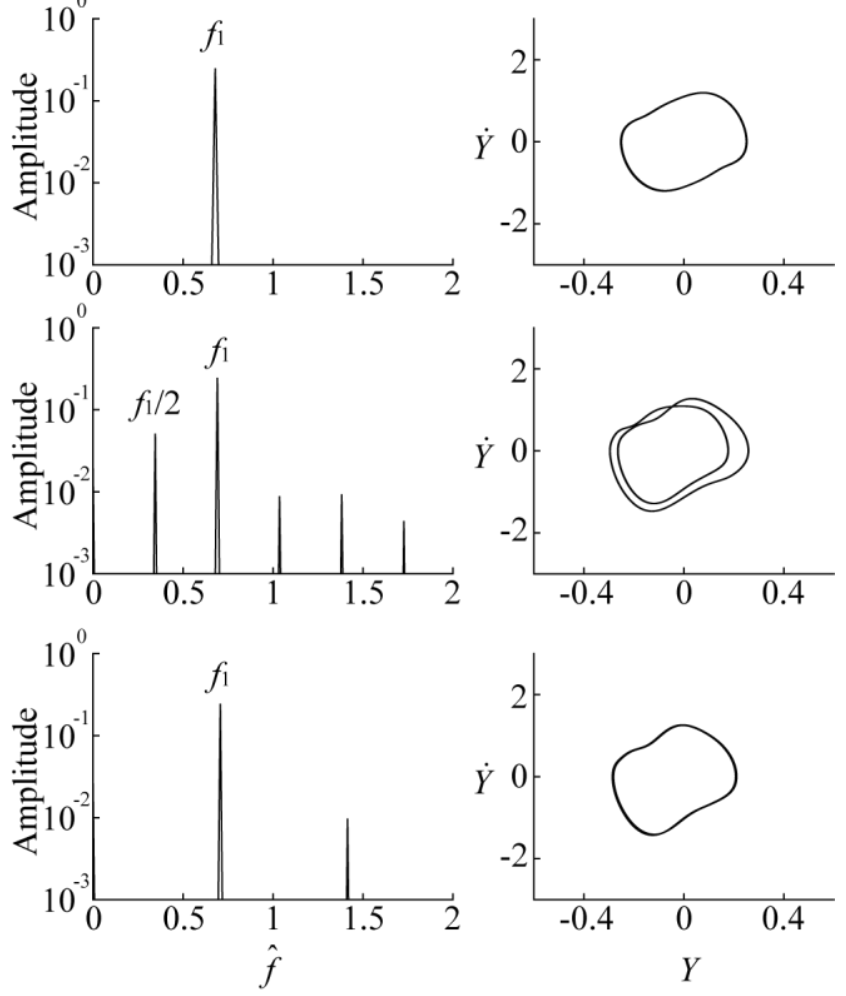

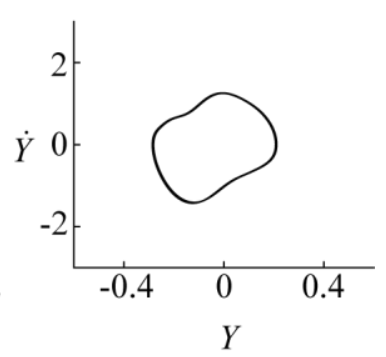

Fig. 12. Transitions from a symmetrical periodic state to an asymmetrical periodic state via a period-doubling bifurcation. For all the cases $M^{*}=1, U^{*}$ is (a) 32.3 , (b) 33.3, (c) 34.1. Left column: time variation of the tail displacement. Middle column: FFT analysis of the tail displacement. Right column: phase plot of the tail velocity in the cross-stream direction against the tail displacement in the cross-stream direction
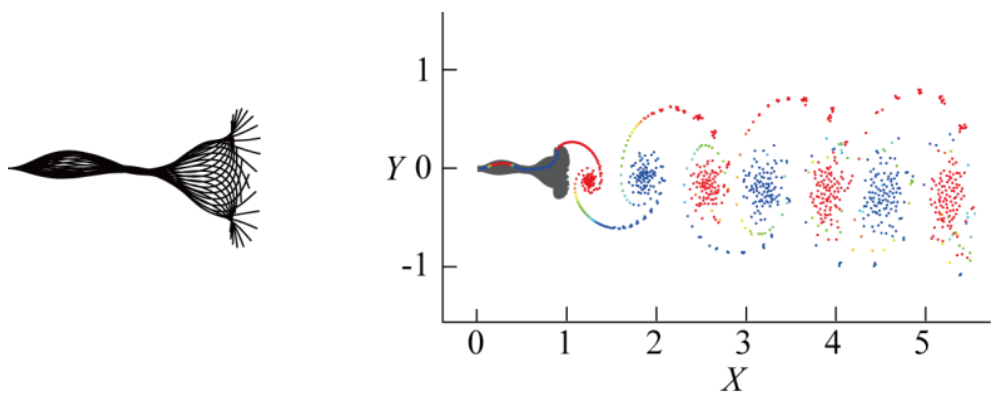

Fig. 13. The snapshot of the flag (left) and the wake structure (right) of the asymmetrical periodic state for $M^{*}=1$ and $U^{*}=34.1$.

\subsection{Transitions from a periodic state to a chaotic state}

Quasi-periodic bifurcations are observed when the system transitions from a 
periodic state to a chaotic state (from region $\mathrm{d}$ to region $\mathrm{f}$, from region $\mathrm{j}$ to region $\mathrm{l}$ ). One of these transitions from region $\mathrm{j}$ to region 1 is shown in Fig. 14. Fig. 14a shows the periodic asymmetrical flapping state when $U^{*}=34.1$ (in region $\mathrm{j}$ ). When $U^{*}$ is increased, quasi-period bifurcations are observed in region $\mathrm{k}$. As shown in the middle column of Fig. 14b, the second basic frequency $f_{2}$ appears when $U^{*}=37.3$. When $U^{*}$ is increased to 41.5 (still in region k), as shown in the middle column of Fig. 14c, the third basic frequency $f_{3}$ appears. When $U^{*}$ is increased to 50 (in region 1), as shown in Fig. 14d, chaotic oscillations are observed. The time variation of the tail displacement shown in the left column of Fig. 14d is irregular, and the spectrum of the FFT analysis result shown in the middle column of Fig. 14d covers a range.

(a)
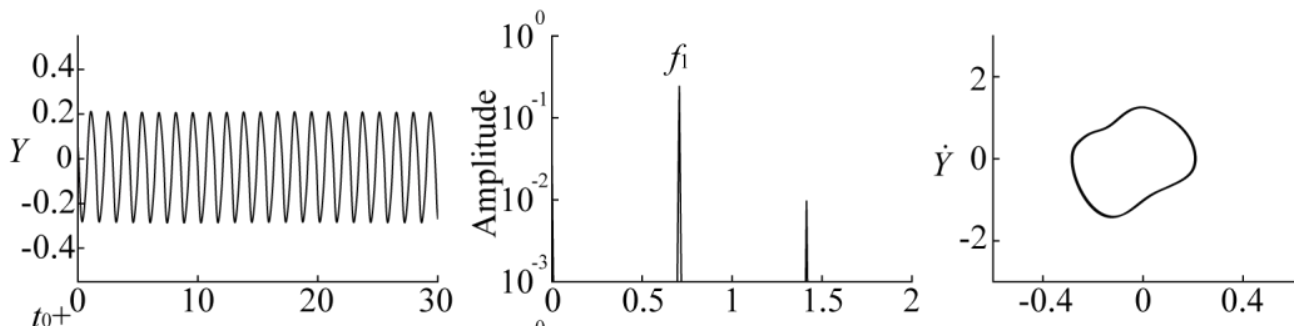

(b)
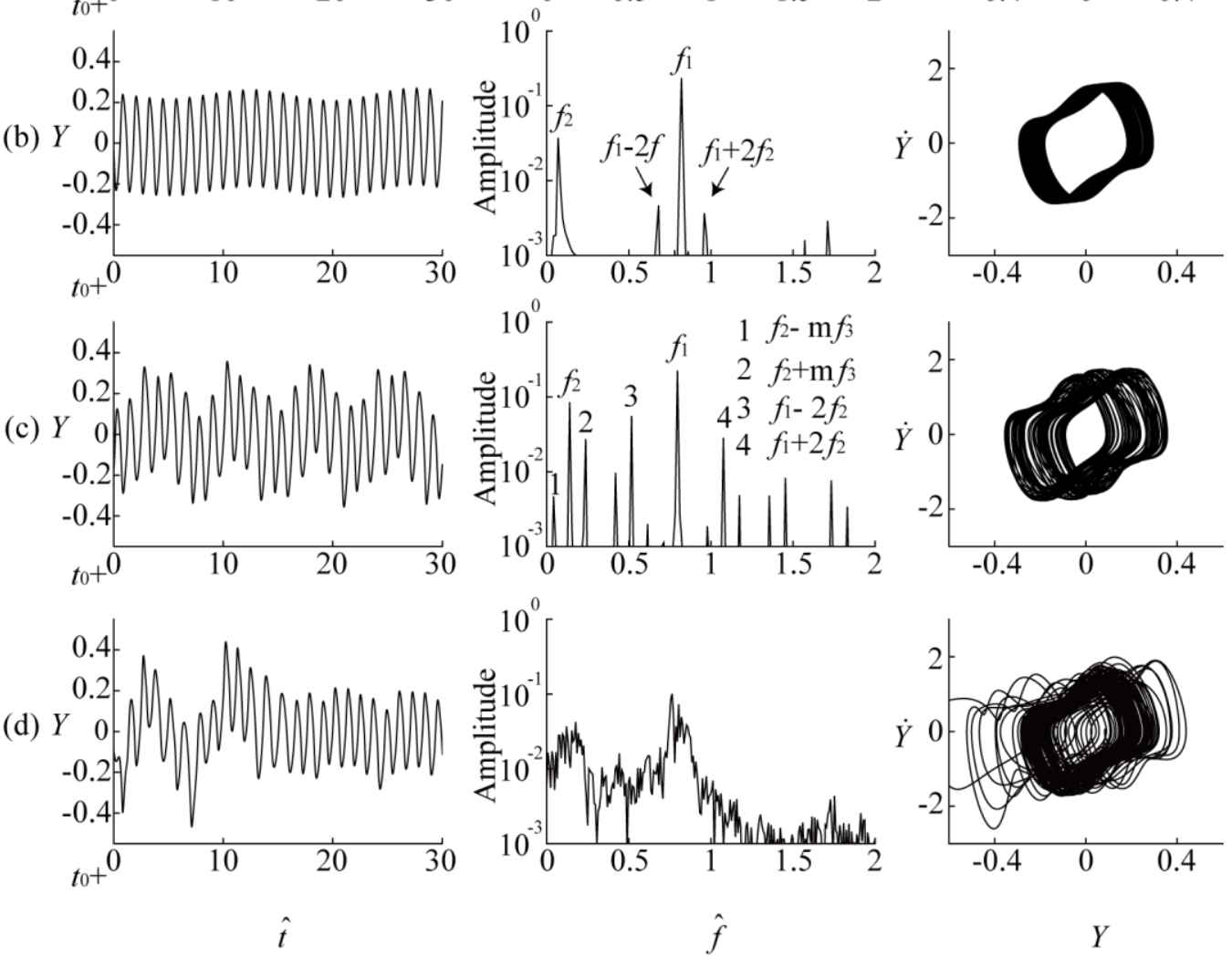

Fig. 14. Transitions from a periodic state to a chaotic state via quasi-periodic bifurcations. For all the cases $M^{*}=1, U^{*}$ is (a) 34.1 , (b) 37.3 , (c) 41.5 , (d) 50 . Left 
column: time variation of the tail displacement. Middle column: FFT analysis of the tail displacement. Right column: phase plot of the tail velocity in the cross-stream direction against the tail displacement in the cross-stream direction.

\section{Conclusion}

A two-dimensional aeroelastic model of a flexible flag immersed in inviscid flow was developed. The system is governed by a structure-to-fluid mass ratio $M^{*}$ and a dimensionless incoming flow velocity $U^{*}$. By fixing $M^{*}$ at unity and increasing $U^{*}$, the transition route from a static steady state to a chaotic state is explored. Five single-frequency periodic flapping states are identified along the route, including four symmetrical states and one asymmetrical oscillation state. For the four symmetrical periodic states, the frequency of the flag increases with the increase of $U^{*}$, and the drag force on the flag changes linearly with the Strouhal number. Chaotic states are observed when $U^{*}$ is relatively large. Three chaotic windows are observed along the route. The system transitions from one periodic state to another periodic state via period-doubling bifurcations or quasi-periodic bifurcations, and it transitions from a periodic state to a chaotic state via quasi-periodic bifurcations.

This work was supported by the NSFC (Grant Nos. 10832010 and 11002138), the Innovation Project of CAS (Grant No. KJCX2-YW-L05), and the K. C. Wong Education Foundation.

\section{Reference}

Ait Abderrahmane, H., Païdoussis, M.P., Fayed, M., Ng H.D., 2011. Flapping dynamics of a flexible filament. Physical Review E, 84, 066604-1-8.

Ait Abderrahmane, H., Païdoussis, M.P., Ng H.D., 2012. Nonlinear dynamics of silk and Mylar flags flapping in axial flow. Journal of Wind Engineering and Industrial Aerodynamics 107-108, 225-236.

Alben, S., 2008. Optimal flexibility of a flapping appendage in an inviscid fluid. Journal of Fluid Mechanics 614, 355-380.

Alben, S., 2012. The attraction between a flexible filament and a point vortex. Journal of Fluid Mechanics 697, 481-503.

Alben, S., Shelley, M.J., 2008. Flapping states of a flag in an inviscid fluid: bistability and the transition to chaos. Physical Review Letters 100, 074301-1-4.

Alben, S., Shelley, M., Zhang, J., 2002. Drag reduction through self-similar bending 
of a flexible body. Nature 420,479-481.

Aurégan, Y., Depollier, C., 1995. Snoring: linear stability analysis and in-vitro experiments. Journal of Sound and Vibration 188(1), 39-54.

Connell B.S.H., 2006. Numerical investigation of the flow-body interaction of thin flexible foils and ambient flow. Ph.D. thesis, Massachusetts Institute of Technology, Cambridge, MA.

Connell, B.S.H., Yue, D.K.P., 2007. Flapping dynamics of a flag in a uniform stream. Journal of Fluid Mechanics 581, 33-67.

Datta, S.K., Gottenberg, W.G., 1975. Instability of an elastic strip hanging in an airstream. Journal of Applied Mechanics 42, 195-198.

Doaré, O., Sauzade, M., Eloy, C., 2011. Flutter of an elastic plate in a channel flow: confinement and finite-size effects. Journal of Fluids and Structures 27, 76-88.

Eloy, C., Kofman, N., Schouveiler, L., 2012. The origin of hysteresis in the flag instability. Journal of Fluid Mechanics 691, 583-593.

Eloy, C., Lagrange, R., Souilliez, C., Schouveiler, L., 2008. Aeroelastic instability of cantilevered flexible plates in uniform flow. Journal of Fluid Mechanics 611, 97-106.

Eloy, C., Souilliez, C., Schouveiler, L., 2007. Flutter of a rectangular plate. Journal of Fluids and Structures 23, 904-919.

Gobat, J. I., Grosenbaugh, M.A., 2006. Time-domain numerical simulation of ocean cable structures. Ocean Engineering 33, 1373-1400.

Hess, J.L., 1990. Panel methods in computational fluid dynamics. Annual Review of Fluid Mechanics 22, 255-274.

Huang, L., 1995. Flutter of cantilevered plates in axial flow. Journal of Fluids and Structures 9, 127-147.

Huang, W.X., Shin, S.J., Sung, H.J., 2007. Simulation of flexible filaments in a uniform flow by the immersed boundary method. Journal of Computational Physics 226, 2206-2228.

Jia, L.B., Li, F., Yin, X.Z., Yin X.Y., 2007. Coupling modes between two flapping filaments. Journal of Fluid Mechanics 581, 199-220.

Katz, J., Plotkin, A., 2001. Low-speed aerodynamics. Cambridge University Press, Cambridge.

Krasny R., 1986. A study of singularity formation in a vortex sheet by the point-vortex approximation. Journal of Fluid Mechanics 167, 65-93.

Michelin, S., Llewellyn Smith, S.G., Glover, B.J., 2008. Vortex shedding model of a flapping flag. Journal of Fluid Mechanics 617, 1-10.

Pang, Z., Jia, L.B., Yin, X.Z., 2010. Flutter instability of rectangle and trapezoid flags in uniform flow. Physics of Fluids 22, 121701-1-4.

Schouveiler, L., Eloy, C., 2009. Coupled flutter of parallel plates. Physics of Fluids 21, 081703-1-4. 
Shelley, M., Vandenberghe, N., Zhang, J., 2005. Heavy flags undergo spontaneous oscillation in flowing water. Physical Review Letters 94, 094302-1-4.

Taneda, S., 1968. Waving motions of flags. Journal of the Physical Society of Japan 24(2), 392-401.

Tang, L., Païdoussis, M.P., 2007. On the instability and the post-critical behavior of two-dimensional cantilevered flexible plates in axial flow. Journal of Sound and Vibration 305, 97-115.

Tang, L., Païdoussis, M.P., Jiang, J., 2009a. The dynamics of variants of two-dimensional cantilevered flexible plates in axial flow. Journal of Sound and Vibration 323, 214-231.

Tang, L., Païdoussis, M.P., Jiang, J., 2009b. Cantilevered flexible plates in axial flow: energy transfer and the concept of flutter-mill. Journal of Sound and Vibration 326, 263-276.

Rodgers, J.L., Nicewander W.A., 1988. Thirteen ways to look at the correlation coefficient. The American Statistician 42(1), 59-66.

Ristroph, L., Zhang, J., 2008. Anomalous hydrodynamic drafting of interacting flapping flags. Physical Review Letters 101, 194502-1-4.

Wang, S.Y., Yin, X.Z., 2010. A numerical method to simulate the coupled oscillations of flexible structures in flowing fluid. Chinese Science Bulletin 55(34), 3880-3888.

Watanabe, Y., Isogai, K., Suzuki, S., Sugihara, M., 2002a. A theoretical study of paper flutter. Journal of Fluids and Structures 16(4), 543-560.

Watanabe, Y., Suzuki, S., Sugihara M., Sueoka, Y., 2002b. An experimental study of paper flutter. Journal of Fluids and Structures 16(4), 529-542.

Ypma, T.J., 1995. Historical development of the Newton-Raphson method. SIAM Review 37(4), 531-551.

Zhang, J., Childress, S., Libchaber, A., Shelley, M., 2000. Flexible filaments in a flowing soap film as a model for one-dimensional flags in a two-dimensional wind. Nature 408, 835-839.

Zhang, J., Liu N.S., Lu X.Y., 2009. Route to a chaotic state in fluid flow past an inclined flat plate. Physical Review E 79, 045306-1-4.

Zhu, L., Peskin, C.S., 2002. Simulation of a flapping flexible filament in a flowing soap film by the immersed boundary method. Journal of Computational Physics $179,452-468$. 